\title{
Changing Health Behaviors through Social and Physical Context Awareness
}

\author{
Guanling Chen, ${ }^{1,2}$ Xiang Ding, ${ }^{1}$ Ke Huang, ${ }^{1} \mathrm{Xu}$ Ye, ${ }^{1}$ Chunhui Zhang ${ }^{1}$ \\ ${ }^{1}$ Department of Computer Science, University of Massachusetts Lowell, USA \\ ${ }^{2}$ State Key Laboratory of Novel Software Technology, Nanjing University, China \\ \{glchen, xding, khuang, xye, czhang\}@cs.uml.edu
}

\begin{abstract}
The single greatest opportunity to improve health and reduce premature death lies in personal behavior. While technology-based behavior intervention has been around for many years, the emerging smartphone and wearable sensing technology brings great promise to push health behavior change further by inferring and predicting real-time behavior occurrence and its context. In this paper, we envision how social and physical context awareness could sustain behavior change motivation and assist health habit formation. We describe our preliminary work that supports this vision and outline the research challenges to be addressed.
\end{abstract}

Keywords-behavior change; behavioral health; behavior sensing; activity recognition; smartphone sensing; context awareness

\section{INTRODUCTION}

Health is influenced by factors in five domains - genetics, social circumstances, environmental exposures, behavioral patterns, and health care. When it comes to reducing early death, medical care has a relatively minor role. In fact, behavioral causes account for nearly $40 \%$ of all deaths in the United States. Although there has been disagreement over the actual number of deaths that can be attributed to the combination of obesity and physical inactivity, it is clear that this pair of factors and smoking are the top two behavioral causes of premature death [1].

Indeed many chronic diseases are caused by unhealthy lifestyles, such as irregular eating, sleeping disorders, addictive substance abuse and lack of exercise. The World Health Organization reported that being overweight and/or obese is one of the leading risks of global deaths. At least 2.8 million adults die each year as a result of being overweight or obese. In addition, a considerable proportion of diabetes, heart disease and certain cancers are attributable to overweight and obesity. ${ }^{1}$ Additionally, tobacco kills nearly 6 million people each year. More than five million of those deaths are the result of direct tobacco use, while more than 600,000 is the result of nonsmokers being exposed to second-hand smoke. Unless urgent action is taken, the annual death toll could rise to more than eight million by $20300^{2}$

There are a variety of behavior theories that can be used by technology-based intervention designers, that would help to

\footnotetext{
1 http://www.who.int/mediacentre/factsheets/fs311/en/

2 http://www.who.int/mediacentre/factsheets/fs339/en/
}

inform which functionality to support, and how to implement such functionality; to guide evaluation strategies and help interpret findings; and to select target users to whom the interventions are more likely applicable [2]. In this paper, our intention is not to survey how constructs from different behavior theories can be combined for a specific intervention, as there seems to be no uniform strategy to do so. Instead, we focus our discussion on a more pragmatic behavior model, proposed by BJ Fogg [3], that complements existing behavior theories but has tangible elements. We argue that these elements can particularly be addressed by social and physical context awareness through advanced smartphone and wearable sensing algorithms [4].

The Fogg Behavior Model (FBM) includes three ingredients required to initiate any and all behaviors: (1) the user must have sufficient motivation; (2) the user must have the ability to complete the desired action; and (3) a trigger must be present to activate the behavior [3.] According to the FBM, all three elements must exist to create a desired behavior, and at least one element must be absent to prevent a unwanted behavior. Fogg identifies three types of motivations for persuasive design: pleasure/pain, hope/fear, and social acceptance or rejection. Abilities mean the desired behavior must be easy to perform. For example, asking a person with a sedentary lifestyle to complete 10,000 steps as a daily goal may well exceed that person's ability. Finally, three types of triggers are listed as sparks (to boost motivation,) facilitators (to make it easy to take action,) and signals (to remind to take action.)

In this position paper, we focus on technology approaches to implement this model for health behavior changes. For the motivational aspect, we emphasize social contexts that influence human behaviors. We identify two types of social conformity and survey health behavior propagation studies in both offline and online social networks (Section II.) For behavior triggers, we emphasize physical context that can be automatically recognized by smartphone or wearable sensors. We discuss how physical cues are important to form health habits by conditioning users for automatic responses. We then survey several emerging techniques to detect high-level physical cues for both external and internal triggers (Section III.) We skip the discussion of the ability element in FBM model as it is application specific. Finally, we outline important research challenges to deliver successful behavior change interventions using social and physical context awareness (Section IV.) 


\section{SOCIAL INFLUENCE}

The behavioral construct of social influence suggests that health-related behavior is influenced by a person's social context. The scope of behavioral social context is rather broad, ranging from individual peers or family members with whom the person interacts regularly, to a larger social environment, such as the neighborhood in which the person lives. The normative social context represents an individual's perceptions about a behavior's acceptability (e.g., smoking, ) derived from explicit communication from network members (e.g. overt pressure to smoke, ) or from implicit observation of behavior(s) of peers (e.g. perceiving typical smokers as cool or popular) or from mass media such as TV or movies (e.g., perceiving smoking as frequent in the population.) This normative social influence reflects that a person conforms to be liked or accepted by others.

Another interesting model of social influence is social proof, also known as informational social influence, which is a psychological phenomenon where people assume the actions of others as correct behavior for a given situation. This effect is most prominent in social situations where the appropriate mode of behavior is ambiguous. A person thus makes an assumption that the people surrounding him/her possess more knowledge about the situation, so their actions are appropriate. While social proof is rationally motivated by considering information possessed by others, it is possible for large groups to converge quickly upon a single choice, either correct or mistaken, that is grounded in very little information. For instance, restaurant menus featuring "popular" dishes may be ordered frequently because people consider popularity as a social proof, despite the concern that the dishes may not be healthy. This may even lead to herd behavior when someone in the group orders these dishes. Similarly, a large group of colleagues who take a walk after lunch may start to draw in more people due to the social proof phenomenon. This kind of conformity reflects that a person is looking to others for cues concerning the correct behavior.

Both types of conformity have fundamental implications on long-term health behaviors and outcomes as our society is organized through social networks. For instance, network phenomena appear to be relevant to the biologic and behavioral trait of obesity, and obesity appears to spread through social ties as evidenced by the following studies.

Christakis and Fowler evaluated the spread of obesity in a densely interconnected social network of 12,067 people assessed over 32 years (from 1971 to 2003,) as part of the Framingham Heart Study [5]. They used longitudinal statistical models to examine whether weight gain in one person was associated with weight gain in his or her friends, siblings, spouse, and neighbors. Their analysis results include: a person's chances of becoming obese increased by $57 \%$ if he or she had a friend who became obese in a given interval; if one adult sibling became obese, the chance that the other adult sibling would become obese increased by $40 \%$; if one spouse became obese, the likelihood that the other spouse would become obese increased by $37 \%$. These effects, however, were not seen among neighbors in the immediate geographic location. While the geographic distance did not correlate with the relative increase in probability of obesity, the social distance played a stronger role in the spread of behaviors or norms associated with obesity. The risk of obesity among peers who were connected to an obese person was about $45 \%$, $20 \%$, and $10 \%$ higher in the observed network than in a random network, for one, two and three degrees of separation, respectively. By the fourth degree of separation, there was no excess relationship between a person's obesity and the connected peer's obesity. Hence, the reach of obesity in this particular social network was three degrees.

Christakis and Fowler's study focused on an offline realworld social network, with self-reported friendship. On the other hand, we conducted a study [6] of an online social network extracted from a weight-loss community, FatSecret, which allows its members to record diaries of food and exercise. Its members are also encouraged to record their weight to track the progress towards weight loss goals. FatSecret members can add each other as friends by following a request and accept process. A user sends out a friendship request to another user, and they become friends if the request is accepted. Like many other online social networks, such as Facebook, the friendships in FatSecret are symmetric. FatSecret users can adjust their privacy settings to share their weight history and diet diaries (food and exercise) with all other users, with their friends only, or with nobody (private mode.) FatSecret engages its users by sending out emails periodically (every two weeks, but adjustable) to remind them to weigh-in (updating their weight on the website.) The reminder email also informs the users about friends' recent performance, like how much weight friends have lost (or gained) every week.

Over the course of about five months in 2010, we collected data from 107,907 users of the FatSecret website. On average, a user only recorded 1.76 weigh-ins (self-reporting current weight) during three months. Most users recorded less than 15 weigh-ins, however, one person recorded 106 weigh-ins. The distribution of the weigh-in intervals fitted better with an exponential distribution. This suggests that the users' weigh-in activities, at least for the FatSecret users during our study, followed a Poisson process so that the consecutive weigh-ins followed each other at relatively regular time intervals, without many long waiting times, unlike Pareto distributions. We observed that the average number of friends, or the network density of the social graph, decreased over time, from February 20th where it was 2.26, to 1.82 on March 25th, then to 1.58 on April 23th, and again to 1.45 on May 20th.

We conducted a correlated analysis and found that a person's weight loss performance increased with the number of weight-ins, suggesting engagement with the service does help to achieve weight loss goals due to amplified awareness and motivation. We also found weight loss performance increased with a person's number of friends, though the correlation seemed to peak when the person had 8 friends. These results suggest that a possible goal of the service could 
be to quickly have a new user create many friends on the site, a tactic often adopted by modern social networks (e.g. Facebook), which increases engagement and ultimately leads to better weight loss outcomes. In addition, we found that the influence of the weight loss success can propagate to the fourth degree of separation [6.] Note the reach of obesity was three degrees of separation in Christakis and Fowler's study of an offline social network [5,] which means the social influence cascades further due to less constraints of information flow in an online environment.

While it is relatively easy to monitor online social networks, offline social interactions are hard to detect in high fidelity (the social network in the Christakis and Fowler's study was tracked manually.) Fortunately, the increased pervasiveness of smartphones presents an opportunity to infer social relationships using observational data from smartphones. In MIT's Reality Mining project [7], over the course of nine months, researchers collected data from 94 subjects using smartphones preinstalled with logging software that recorded data about call logs, Bluetooth devices in proximity of approximately five meters, cell tower IDs, application usage, and phone status. Researchers also collected self-report relational data from each individual, where subjects were asked about their proximity to, and friendship with, others. Subjects were also asked about their satisfaction with their work group. The experiment showed that it was possible to accurately infer $95 \%$ of friendships based on the observational data alone. While the inferred friendships had more distinct temporal and spatial patterns than the self-reported friendships, their predictive power over individual outcome, such as job satisfaction, was almost identical. These results validated the measure of friendship based on observational smartphone data.

We need to acknowledge that social influence is a double edged sword, as motivated people can find social support from a like-minded group to change behaviors, while social proof in ambiguous situations and social pressure to be liked or accepted, may conform people to undesired behaviors. On the other hand, having people commit to public or anonymous groups of strangers, for the goal of behavior change (e.g. quit smoking or lose weight) is a powerful social motivator. People are now held accountable for their actions and they are motivated to follow through to keep their pride intact.

\section{PhysicAl CUeS}

Recent literature demonstrates the relevance of habitformation principles to behavior health. Habit-formation advice, paired with a 'small changes' approach, has been tested as a behavior change strategy [8]. Habits are automatic responses to frequently encountered contexts triggered by either external or internal cues. Habits are essential because they free our conscious minds from handling mundane details, for everything from thinking about how to brush teeth in the morning to how to drive a car to work. Habits form when the brain takes a shortcut and stops actively deliberating over what to do next. Without habits, our conscious minds would be overloaded with the details of daily decisions. Researchers estimate that roughly half of our daily lives are spent executing habits and other intuitive behaviors, and not consciously thinking about what we are doing. Thus, it is desirable to assist people to form healthy habits with minimal cognitive load making the desired health outcomes much easier to maintain.

Recent studies suggest that performance contexts, with minimal influence of goals, automatically trigger mental representations of associated habitual responses [9]. Thus, strong habits - although perceived to be purposeful and goaldependent - are actually influenced by recurring triggers in the performance context. Based on this theory, Lally et al. conducted a weight-loss intervention using habit formation advice and simple recommendations for eating and activity behaviors promoting negative energy balance [10]. This simple intervention produced clinically significant results: at 8 weeks, people in the intervention condition had lost significantly more weight (mean $=2.0 \mathrm{~kg}$ ) than those in the control condition $(0.4 \mathrm{~kg})$ and at 32 weeks, those who remained in the study had lost an average of $3.8 \mathrm{~kg}$, with $54 \%$ losing $5 \%$ or more of their body weight. However, the formation of strong habits can take from 18 to 254 days depending on habit complexity and individual differences [11].

We believe that modern technology, particularly the pervasive availability of sensor-rich smartphones and emerging wearable devices, has reached a point to effectively help detect the physical cues that trigger the automatic response of habits. Thus conceivably, we could alert people when the cues present to assist forming new habits (e.g. taking a walk after lunch,) or to intervene just in time to stop the actions of old habits (e.g. smoking while watching TV.) Note that physical cues can be either external triggers (e.g. morning alarms) or internal ones (e.g. being hungry or bored.) Both signals are just as effective in influencing our daily behavior. With technology-assisted cue conditioning, we expect to shorten the habit process and make stronger associative responses.

The location of people is a key context providing rich semantics to their activities. Arriving and leaving a place can also serve as physical cues to trigger desired actions. For example, a habit can be conditioned to drink a glass of water when arriving at workplace in the morning, or to take a short walk when leaving the office in the afternoon. Similarly, people may also want to form a habit of taking vitamins when entering the kitchen after getting up in the morning. Smartphone sensing can detect these events and remind users to perform habit routines under such contexts, which facilitates the habit formation process. After being repeated enough times, the habit will become stable and automatic without the prompts.

Continuously tracking a person's location, particularly at the room level accuracy, is not a trivial task. While modern smartphones all have GPS sensors, they consume significant power and do not work indoors. On the other hand, we observed that people spend most of their time indoors with their phones stationary (e.g. on the desk.) Thus, we propose a tracking system, MVPTrack, that monitors a smartphone's motion based on accelerometer's readings to determine when to start localization [12]. Namely, we only need to find a location when a person has arrived at a place, which is 
identified using Wi-Fi signatures (a list of nearby APs and their signal strength profile.) Thus, MVPTrack can effectively follow a person's walking, driving, and stationary activities throughout his/her day without consuming much battery power, since the accelerometer is a power-efficient sensor (often one or two magnitudes less than using a GPS sensor.) Our experiments showed that MVPTrack consumed about $60 \%$ less energy compared with a state-of-art Wi-Fi location tracking approach (e.g. SensLoc [13].) Over typical days, MVPTrack used less than $5 \%$ overall battery power and was not noticeable to users.

Sleep is essential for a person's health and wellbeing, and health habits can be formed around bedtime and wakeup time (e.g. practice meditation before sleep and do 10 push-ups after getting up.) Thus, it is important to detect sleep relevant cues. We propose a smartphone-based approach that provides unobtrusive and continuous monitoring of bedtime and sleep duration [14]. A context-aware inference engine predicts whether a user is asleep or awake using a variety of contextual signals collected from smartphone sensors. Our study using an 10 -week data set from 11 participants showed that the supervised learning algorithms could achieve reasonable detection accuracy. The average bedtime error was less than 15 minutes and average sleep duration error was about 30 minutes, which was better than the state-of-art detection methods. We further propose an unsupervised learning approach that does not require users to manually train the detection system, with accuracy results only slightly worse than supervised approaches. To the best of our knowledge, this is the first study of an unsupervised approach for sleep monitoring, making it especially attractive for long-term passive sleep monitoring of populations who are not willing or able to train the supervised learning algorithms.

There are, of course, a variety of other user activities that can be used as physical cues to condition healthy habits. For instance, some people may choose to form a habit of eating fruits when watching TV [10]. It is possible to detect TVwatching activity using microphone and camera sensors on modern smartphones [15]. User's eating activity can also serve as a physical cue. For example, a habit of taking a walk or drinking water can be conditioned after eating lunch. Given the unique hand and wrist motion when eating, it is possible to detect eating activity with a reasonable accuracy using a wristworn motion sensor. Our experiments based on an off-the-shelf smart watch showed that decision tree classification achieved F1-measure of $92 \%$ accuracy using only time-domain features from 3 -axis accelerometer data [16].

External triggers present to users through sensory stimuli, such as eating meals and watching TV. Internal triggers cannot been seen, touched, or heard, as they manifest automatically in a person's mind. Emotions, particularly negative ones, are powerful internal triggers and greatly influence our daily routines. Feelings of boredom, loneliness, frustration, confusion, and stress, typically prompt an almost instantaneous, and often mindless, response to quell the negative sensation. For example, people check Facebook or Twitter when they feel bored, or smoke cigarettes when they feel stressed.
Interestingly, smartphone sensing can be used to detect these kinds of internal cues that are typically invisible. StressSense is a system to unobtrusively recognize stress from human voice using smartphones. The classifier can robustly identify stress across multiple individuals in diverse acoustic environments. Using model adaptation, StressSense achieved $81 \%$ and $76 \%$ accuracy for indoor and outdoor environments, respectively [17]. MoodScope is another mobile system that infers a user's mood based on the user's location and application usage patterns. The experiments showed that MoodScope could statistically infer a user's daily mood with an initial accuracy of $66 \%$, which gradually improved to an accuracy of $93 \%$ after a two-month personalized training period [18]. In addition, commercial products are becoming available that can directly measure a person's internal state, such as stress level, through wearable devices. Given these promising results, it may be possible to directly leverage internal triggers as physical cues to condition health habits.

\section{RESEARCH CHALLENGES}

In this paper, we have discussed behavior changes by focusing on: 1) motivation through social influence, and 2) detecting physical cues to assist forming health habits. Tying these two aspects together to successfully deliver behavior change interventions in a real-world setting is still an unexplored area despite increased attention from researchers and practitioners. In this section, we identify several research challenges to hopefully generate discussions and to receive feedback from the community.

To sustain behavior change motivation, a person might join a social group of people who have similar situations. For example, in the FatSecret study we found that members who had similar weight and weight loss goals most likely formed friendships [6]. Other online health communities, such as PatientsLikeMe, have adopted the same principles. Representatives from offline counterparts include Weight Watcher and various "meet-up" and social clubs. By joining these social networks, behavior change may be sustained as people find mutual support.

One interesting question is how to quickly engage new members after they have joined a group. A solution is getting new members to connect with influential existing users, an approach adopted by many online social networks. The recommendation for users to connect could be based on similarity of lifestyle, background, interests, and particularly health conditions. In an early study, we predicted online friendships using a three-dimension model considering mobility pattern, social distance, and profile interests. Our experimental results using a real social network of 18,951 active users showed the predictive model achieved $48.5 \%$ accuracy when making the top 100 recommendations [19]. We expect this kind of model could be adapted to connect and engage health-oriented social network services as well. In particular, the recommended algorithms needed to influence the social network topology to maximize the impact of successful health outcomes. For example, it might be desirable 
to have a dense short-diameter network (e.g. each pair of users are separated no more than 4 degrees [6].)

While people mostly find positive feedback from online communities, they may experience negative influences from offline social interactions, particularly with people not motivated for behavior changes, as evidenced by Christakis and Fowler's study [5]. Smartphones can be used to reconstruct the offline social networks using observational data (e.g. Bluetooth proximity) [7]. Thus, it is an interesting question whether we could identify the key individuals in the network from targeted behavior changes. By converting and motivating these people toward positive health behaviors, we might efficiently curb the bad influence and effectively promote desired outcomes.

For health habit formation, we envision that smartphones could be used to automatically detect physical cues (external or internal) and prompt users into desired actions. After many repetitions, strong habits might form and could sustain automatically without further prompts. While users may explicitly choose the cues to condition the desired habits, it may be better for algorithms to analyze the traces collected from smartphone and wearable sensors, to intelligently choose physical cues that would work best for an individual. This is desirable as strong habits responding to frequent cues occur in stable contexts. This requirement imposes significant technical challenges on energy-efficient sensing, high-level activity recognition, fine-grained life logging, and smart analysis algorithms.

In previous discussions, we have focused on creating new health habits. On the other hand, it is very difficult to break existing unhealthy habits due to their automatic nature rather than conscious decision. For example, two-thirds of alcoholics who complete a rehabilitation program will pick up their old habits of drinking within one year. Research shows that nearly everyone who loses weight on a diet gains back the pounds within two years. To make things worse, these people are usually not aware as to what physical cues have triggered their bad habits [20]. For example, a person who smoked several times at a certain place, may have conditioned himself to smoke when in that specific place again, without being aware of the stimulus (experience reinforcement.)

Thus a research challenge is whether it is possible to detect unknown cues that trigger bad habits automatically. On the other hand, we can detect undesired behaviors, such as smoking or overeating, by using wrist-worn sensors [16] as the body-area sensor network [20] becomes mainstream. These behaviors could be correlated to all other traces collected by smartphones regarding environment, activities, and internal states (stress, mood, etc.) With such comprehensive traces, we may be able to detect triggering cues, or at least we may be able to predict smoking or overeating episodes based on contexts without actually identifying exact cues. Once we can establish such predictive models, interventions can be designed to inhibit old habits by distraction, avoidance, or replacement of new routines [21].
Finally, security and privacy are important considerations for the proposed research as personal data are constantly being collected and analyzed. Avancha et al. provide an in-depth survey on related issues [22].

\section{ACKOLWEDGEMENTS}

This work is supported partly by the National Science Foundation under Grant No. 1040725 and No. 1016823.

\section{REFERENCES}

[1] S. A. Schroeder. We Can Do Better - Improving the Health of the American People. N Engl J Med, 357:1221-1228, Sept. 2007.

[2] E. Hekler, P. Klasnja, J. E. Froehlich, and M. Buman. Mind the theoretical gap: interpreting, using, and developing behavioral theory in HCI research. CHI 2013.

[3] BJ Fogg. A Behavior Model for Persuasive Design. Persuasive 2009.

[4] N. D. Lane, E. Miluzzo, H. Lu, D. Peebles, T. Choudhury, A. T. Campbell, A Survey of Mobile Phone Sensing, IEEE Communications Magazine, September, 2010.

[5] N. Christakis and J. Fowler. The Spread of Obesity in a Large Social Network over 32 Years. N Engl J Med 2007;357:370-9.

[6] X. Ma and G. Chen. Understanding Weight Change Behaviors through Online Social Networks. International Journal of Computational Models and Algorithms in Medicine (JCMAM), 2(3):46-69. July 2011.

[7] N. Eagle, A. Pentland, and D. Lazer. Inferring Social Network Structure using Mobile Phone Data. Proceedings of the National Academy of Sciences (PNAS) Vol 106(36), pp. 15274-15278. 2009.

[8] B. Gardner, P. Lally, and J. Wardle. Making health habitual: the psychology of 'habit-formation' and general practice. Br J Gen Pract December 2012 62:664-666.

[9] D. T. Neal, W. Wood, J. S. Labrecque, P. Lally, How do habits guide behavior? Perceived and actual triggers of habits in daily life, Journal of Experimental Social Psychology, 48(2): 492-498, March 2012.

[10] P. Lally, A. Chipperfield, J. Wardle. Healthy habits: Efficacy of simple advice on weight control based on a habit-formation model. Int J Obes (Lond). 2008 Apr;32(4):700-7. Epub 2007 Dec 11.

[11] P. Lally, C. van Jaarsveld, H. Potts and J. Wardle. How are habits formed: Modelling habit formation in the real world. European Journal of Social Psychology 40(8): 998-1009. Oct. 2010.

[12] C. Zhang, K. Huang, and G. Chen. MVPTrack: Energy-Efficient Places and Motion States Tracking. MobiQuitous 2013.

[13] D. H. Kim, Y. Kim, D. Estrin, and M. B. Srivastava, "SensLoc: Sensing everyday places and paths using less energy" SenSys, 2010.

[14] K. Huang, X. Ding, J. Xu, G. Chen. Detecting Irregular Sleep Nights through Unconstrained Smartphone Sensing. Under review, Oct. 2014.

[15] M. Ibrahim, A. Saeed, M. Youssef, K. A. Harras. Unconventional TV Detection using Mobile Devices. UBICOMM 2013.

[16] X. Ye and G. Chen. A Feasibility Study of Eating and Smoking Recognition using Smart Watches. Under review, Oct. 2014.

[17] H. Lu, D. Frauendorfer, M. Rabbi, M.S. Mast, G.T. Chittaranjan, A. Campbell, D. Gatica-Perez, T. Choudhury. StressSense: detecting stress in unconstrained acoustic environments using smartphones. UBICOMP 2012.

[18] R. LiKamWa, Y. Liu, N. Lane, and L. Zhong. MoodScope: building a mood sensor from smartphone usage patterns. MobiSys 2013.

[19] N. Li and G. Chen. Multi-Layer Friendship Modeling for LocationBased Mobile Social Networks. MobiQuitous 2009.

[20] L. Wang, T. Gu, X. Tao, and J. Lv. A Hierarchical Approach to Realtime Activity Recognition in Body Sensor Networks. PMC 8:1, Feb. 2012.

[21] J. M. Quinn, A. M. Pascoe, W. Wood, \& D. T. Neal (2010). Can't help yourself? Monitor those bad habits. Personality and Social Psychology Bulletin, 36, 499-511.

[22] S. Avancha, A. Baxi, and D. Kotz. Privacy in mobile technology for personal healthcare. ACM Computing Survey 45:1, Nov. 2012. 